\title{
Study of different structures of additive genetic groups to increase the efficiency of genetic selection in beef cattle
}

\author{
G.A. Oliveira Junior \\ 2013. Faculdade de Zootecnia e Engenharia de Alimentos, Universidade de \\ São Paulo, Pirassununga, SP, Brasil \\ Master's thesis. Orienting Prof.: Dr. Joanir Pereira Eler \\ DOI http://dx.doi.org/10.4238/2014.January.10.1 \\ Corresponding author: G.A. Oliveira Junior \\ E-mail: gersonjr@gmail.com
}

The structure of genetic groups is a technique that allows animals with unknown paternity to be included in genetic evaluation programs. The ways these groups are formed are still arbitrary, making it important to study different formation strategies for genetic groups, aiming for a suitable framework for the genetic evaluation of seedstock in animal breeding programs. Therefore, the aim of this study was to define the structure of genetic groups suited for genetic evaluations, comparing them in regard to changes in the effective efficiency of the processing of animals with unknown parentage. Data from 116,368 Nellore cattle were analyzed focusing on the traits weaning weight, post-weaning weight, post-weaning weight gain, scrotal circumference at 18 months of age, and visual muscularity score at 18 months of age. Three scenarios were simulated from a database consisting only of complete pedigree animals (control group). The first scenario cut $30 \%$ of sires, the second cut $50 \%$, and the third $70 \%$. The training strategies for additive genetic groups were: the birth farm of the animal with unknown parentage; birth year (CROP) and the concatenation of year of birth and birth farm (CROPFARM). The variance components were calculated for the data bank control program by VCE, and breeding values were predicted using the PEST software, with two structures of models that differ by the inclusion or not of the fixed effect of additive genetic groups. The definition of the structure of genetic group suitable for genetic evaluation of animals was based on the efficiency of selection and comparison of estimated breeding values to the "control" breeding values and the rank of animals, where it was assumed that control animals 
would have the maximum response to selection and any other group of animals would result in a reduction thereof. The results demonstrated that the additive genetic groups provide an improvement in the genetic prediction of animals with unknown paternity. Strategies that provided values closer to those of the control group were CROP and CROPFARM, and although showing similar values, the CROP strategy was superior in selecting the best animals.

Key words: Animal breeding; Correlation; Multiple sire; Unknown paternity; Pedigree 